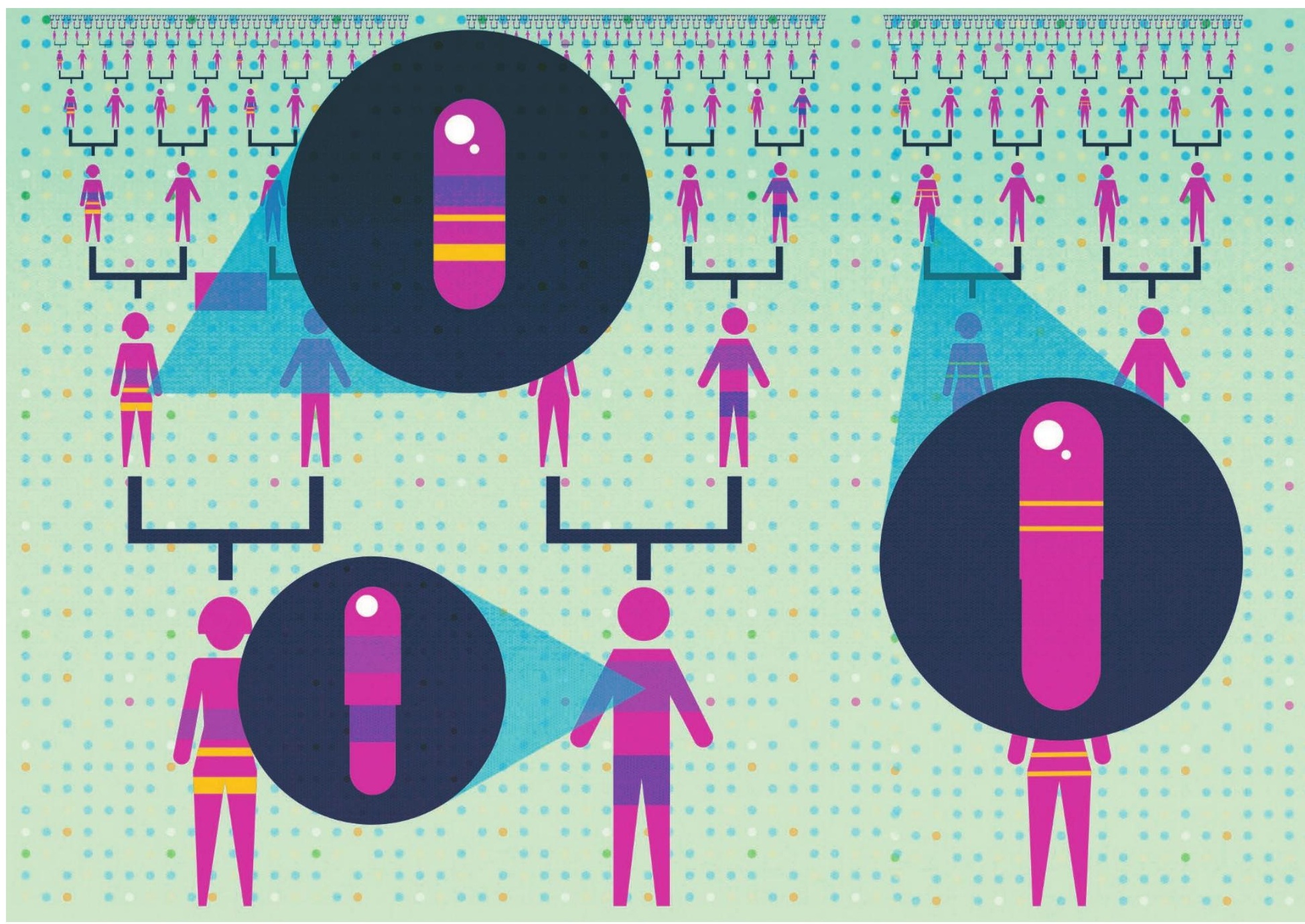

\title{
The right drug for you
}

\section{Personalized prescribing is gaining momentum, but is there enough evidence for it to become standard clinical practice?}

\section{BY LIAM DREW}

$\mathrm{F}$ or ten-year-old leukaemia patient Jason Saunders, the usual chemotherapy was not going to work. Given the standard drug treatment, there was a strong chance that toxic metabolites would build up in his body and make him sick, requiring a break in his therapy that would allow the cancer to return.

This is because Jason is one of the $10 \%$ of Caucasians with a genetic variation that reduces his ability to metabolize thiopurines, the drugs most commonly used to treat acute lymphoblastic leukaemia. Rather than having two high-activity copies of the TPMT gene that produces the enzyme responsible for metabolizing these drugs, Jason has only one. Luckily for him, the doctors at St Jude Children's Research Hospital in Memphis, Tennessee, knew that. When he was diagnosed with cancer, one of the first things his physicians did was take a sample of his blood to assess how he might respond to the drugs. As a result, Jason was given a lower dose of thiopurines than nor$\mathrm{mal}$, and he tolerated the therapy without needing a break. He is now in remission.

Testing leukaemia patients for their TPMT status is one of the most common examples of treatment being tailored to match patients' genetics, so robustly does it predict the likelihood of adverse effects from thiopurines. The test is now mandatory when starting this type of chemotherapy in many places, and it is also recommended before using thiopurines to treat other conditions, such as inflammatory bowel disease and rheumatoid arthritis.

Tests are normally performed only when a drug needs to be prescribed, but Jason's experience was different. Instead of testing only his TPMT status, he was screened for variability across a host of genes involved in various drug responses, as part of the hospital's PGEN4Kids scheme. The results were then incorporated into his health records. If he is ever prescribed another drug for which those genes can predict an adverse response, the information will be presented to his doctor immediately. The choice of which drug to use, and at what dose, 


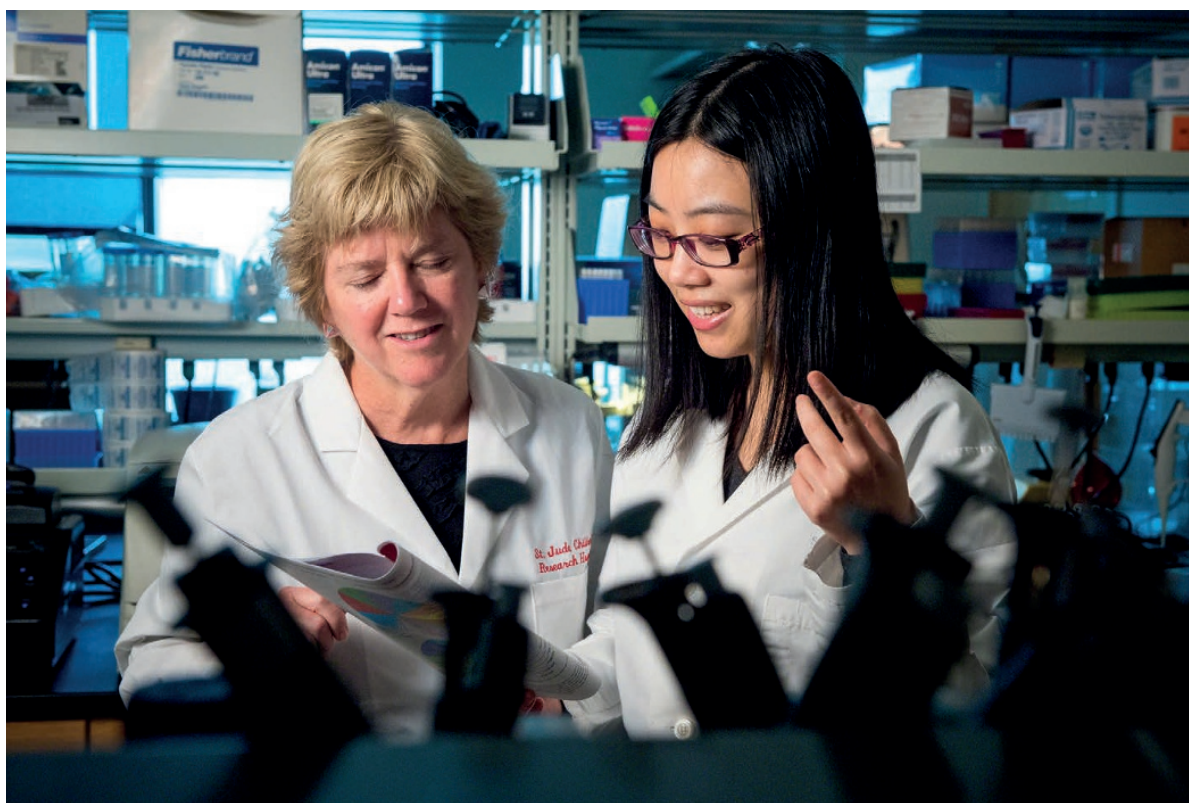

Mary Relling (left) is trying to show that pharmacogenetics can improve outcomes for patients.

and are working through identified genes at a rate of about three or four a year, each of which may relate to multiple drugs.

One recent publication, for example, explains how to prescribe several related antidepressants to patients with common variants of the genes CYP2D6 and CYP2C19, which affect the metabolism of these drugs. It recommends avoiding certain antidepressants in some patients, or reducing the dose by $50 \%$ if they are deemed essential. Relling hopes that St Jude's health records will eventually contain information on all of the genes that CPIC views as actionable.

\section{BURDEN OF PROOF}

The screening programmes being pioneered at leading US hospitals are resolving many of the practical issues that once restricted the clinical use of pharmacogenetics. But for them to become routine elsewhere, health-care funders need to be fully convinced of their value.

"We have a lot of science that is really good. We have a lot of growing infrastructure and growing education," says Scott. "But these programmes that are doing implementation are funded by research grants." The extent to which health-care providers are willing to financially support clinical pharmacogenetics will have a significant effect on its future.

There is a feeling among those in the field that they have done enough: that the science is solid, and that numerous observational studies have shown the value of clinical implementation. Relling says that the scientific case for PGEN4Kids is overwhelming. "It would be unethical to withhold these tests," she says.

Munir Pirmohamed, a clinical pharmacologist at the University of Liverpool, UK, thinks that pharmacogenetic tests are held to unreasonably high standards - a bias he calls "genetic exceptionalism". A patient with kidney failure will

have that taken into account during drug selection and dosing as a matter of course, explains Pirmohamed, but a genetic variation that affects drug metabolism to the same degree is held to a higher standard of proof. "People ignore it. People say you need a randomized controlled trial," he says. "That's unreasonable."

But not everyone is convinced. James Evans, a medical geneticist at the University of North Carolina at Chapel Hill, urges cau-

"We've had
50 years of
research
showing why it
should be done."
tion. "We have to be careful," he says. "We need evidence of benefit before we implement."

The field of pharmacogenetics has not produced compelling evidence by using randomized control trials - the gold standard for evidence-based medicine. Evans has been outspoken about the bloated expectations that surround genomics-based medicine and is blunt about what would best deliver evidence. "We need to use pharmacogenetics and not use pharmacogenetics, and see if there's a benefit," he says. That will entail studies of the sort that showed that one particular type of pharmacogenetics, involving the anticoagulant drug warfarin, was not useful, he adds.

The warfarin case is instructive. The rates at which individuals metabolize this drug vary widely. Break it down too quickly and it will not have the desired effect; too slowly, and the risk of bleeding increases. Levels of the drug in the blood are therefore typically monitored for weeks at the start of treatment, and drug dosages are adjusted accordingly - a process that requires repeated visits to a clinic. Because variants of two genes have large effects on the rate at which warfarin is metabolized, it was widely expected that clinical trials would show that warfarin dosing is improved by screening for patients' genotypes. But trials showed that genetic screening led to no improvement in drug dosing.

Even here, however, some promise remains. A later trial led by Pirmohamed found a small but significant improvement when factors beyond genetics, such as the patient's age, body mass and other drugs they were taking, were considered. Using genetic information in combination with other factors may be better than using it in isolation. "I think there is promise in pharmacogenetics," says Evans, "but it's not going to revolutionize everything we do."

Pirmohamed is now part of a team that has secured US\$17 million from the European Union's Horizon 2020 funding programme to run the sort of large, randomized control trial that people want to see. Ubiquitous Pharmacogenomics will spend 3 years studying the clinical outcomes of 8,000 patients in 7 hospitals in 7 European countries. The trial will use a panel of 13 genes, and patients will be randomly assigned to groups that either do or do not receive pharmacogenetics-led prescription of drugs affected by those genes.

Proponents of pharmacogenetics stress that the outcomes of such trials are not the only results that should influence medical practice. Positive results have been seen in observational studies at hospitals such as St Jude that have nascent pharmacogenetics programmes, and Pirmohamed thinks that data from the UK 100,000 Genomes Project will also strengthen the case for clinical pharmacogenetics.

Relling is confident that pre-emptive clinical genotyping will one day be the norm. "We've had 50 years of research showing why it should be done," she says. But there is no doubt that the unambiguous results of a randomized clinical trial of $H L A-B$ testing before prescribing abacavir to HIV patients in 2008 were pivotal to the test being widely adopted. So the upcoming Ubiquitous Pharmacogenomics trial, which begins next year and should report results in 2020, may have an important role in the future of personalized prescribing.

For Shelley Saunders, whose son Jason is part of St Jude's PGEN4Kids scheme, clinical pharmacogenetics seems to be ready for prime time. The choice to enrol in the programme was "one of the easier decisions we had to make", she says. "I don't know why anyone wouldn't want this."

But as physicians Evans and Pirmohamed are well aware, patients' safety must not be jeopardized by the pursuit of an apparently good idea. As the evidence builds, the use of pharmacogenetics may well become a common sight in hospitals and pharmacies around the world. But medicine is inherently conservative, and solid data are required if clinical practice is to change.

Liam Drew is a science writer based in London, UK. 